\title{
Estudio Empírico del Trabajo Calificado en Chile: Productividad y Remuneraciones
}

\author{
EMPIRICAL STUDY IN QUALIFIED WORK IN CHILE: PRODUCTIVITY AND REMUNERATIONS
}

Guillermo Riquelme Silva', Alberto López Toro², Víctor Erik Olivares Faúndez ${ }^{3}$

1. Investigador Asociado al Centro de Estudios y Gestión Social. Universidad Autónoma de Chile.

2. Dpto. Economía y Administración de Empresas. Universidad de Málaga, España.

3. Profesor Escuela de Psicología, Universidad de Santiago de Chile, USACH.

\section{RESUMEN}

El objetivo del estudio es contrastar las hipótesis más recurrentes en la literatura económica respecto de la productividad y las remuneraciones del trabajo calificado en Chile. Para ello se analiza y evalúa la relación de largo plazo entre las variables productividad y remuneraciones para el trabajo calificado, utilizando series de tiempo que comprenden el período 1990-2009. Los resultados dan cuenta de la estacionariedad de los residuos, lo que implica que las variables no están cointegradas. Por lo que se puede concluir que no existe evidencia empírica, para el caso de Chile, de relaciones de largo plazo entre las variables estudiadas, aunque se observa un aumento significativo de la brecha de ingresos entre los trabajadores cualificados y los que no lo son.

(Riquelme G, López A, Olivares V, 2014. Estudio Empírico del Trabajo Calificado en Chile: Productividad y Remuneraciones. Cienc Trab. May-Ago; 16 [50]: 81-87).

Palabras claves: TRABAJO CALIFICADO, PRODUCTIVIDAD, REMUNERACIONES, CAPITAL HUMANO, EDUCACIÓN SUPERIOR.

\section{ABSTRACT}

The objective of the study is to compare the most frequent hypothesis in economic literature on productivity and wages of skilled labor in Chile. This is analyzed and evaluated the long-term relationship between the variables productivity and wages for skilled labor, using time series comprising 1990-2009. The results show stationarity of waste, which means that the variables are not cointegrated. As can be concluded that there is no empirical evidence for the case of Chile, for long-term relationships between the variables studied, although a significant increase in the income gap between skilled workers and those that are not observed.

Key words: QUALIFIED WORK, PRODUCTIVITY, WAGES, HUMAN CAPITAL, HIGHER EDUCATION.

\section{INTRODUCCIÓN}

La discusión y debate de la educación superior en Chile, sin duda, tiene un ámbito más amplio que la sola "Certificación de la Calidad de la Educación Superior", que es el de la Real Eficiencia y Eficacia, en términos de Productividad para las empresas y de Recompensa para los Trabajadores Calificados, de los Recursos Públicos Invertidos en Educación Superior.* Más aun, cuando estos recursos públicos invertidos en educación persiguen, en gran medida, contribuir a reducir la desigualdad socioeconómica observada en Chile $^{1}$ y converger hacia una equidad social, que es parte integral de la calidad de la educación. ${ }^{2}$

\section{Correspondencia / Correspondence:}

Guillermo Riquelme Silva

Centro de Estudios y Gestión Social del Maule,

Universidad Autónoma de Chile, Sede Talca

5 poniente 1670. Edificio Aulas 5, Primer Piso

e-mail: griquelmes@uautonoma.cl

www.cegestionsocial.cl

Tel.: (+56 71) 2735690

Recibido: 19 de Mayo 2014 / Aceptado: 19 de Julio 2014
En este contexto, el trabajo calificado o capital humano avanzado se releva como uno de los factores productivos más importantes para generar mayor crecimiento y bienestar socioeconómico de un país. Asumiendo que para alcanzar dicha calificación se requiere fundamentalmente de un proceso educativo formal, ya sea de nivel técnico o profesional, que permita proporcionar las competencias necesarias y suficientes para que un individuo pueda desempeñarse productivamente en el campo laboral. Sin por ello desconocer que en la literatura económica existen investigaciones $\mathrm{y}$ análisis teóricos sobre el trabajo calificado, que no necesariamente ha obtenido su calificación a través de una educación formal, sobre todo en lo que se refiere al trabajo en Latinoamérica, como es el caso de los estudios de Giménez y Peñaranda. ${ }^{3,4}$

En cuanto a las crecientes exigencias de la sociedad en el ámbito de la educación superior en Chile, los requerimientos de eficiencia y eficacia han cobrado cada vez más fuerza en la discusión social y política, fundamentalmente por tres grandes aspectos:

1) El aumento progresivo y significativo de los montos considerados en las diferentes partidas presupuestarias referidas a la educación ${ }^{5}$ a partir de la década de los 90. En Chile, a partir del año 1990, se produce un incremento significativo de los recursos financieros asignados a la educación. El gasto público en educación sobre el PIB pasó del 2,4\% en 1990 al 2,7\% en 1995, al 3,9 en el año 2000, al 4,2\% en el 2008 y al 5,4\% el 2012. En los últimos años el gasto público en educación representa alrededor del 20\% del total del gasto público, pasando a ser el 
segundo sector de mayor significancia en el gasto público nacional después de la protección social.

2) Los bajos resultados obtenidos a través de mediciones nacionales e internacionales en materia de Calidad de Educación. De esto da cuenta a nivel nacional los resultados del SIMCE y a nivel internacional los resultados obtenidos en las pruebas TIMSS y PISA.

3) El bajo nivel de productividad a pesar del crecimiento económico experimentado (http://www.ccs.cl/html/publicaciones/publicaciones/2011/Abril/Productividad\%2011-04-11.pdf).

En este nuevo escenario, la rendición de cuenta pública en la educación superior (accountability educacional) se hace cada vez más imprescindible, y que incluye tanto aspectos de gestión educativa como de gestión financiera. En cuanto a la gestión educativa, se contempla el proceso educativo propiamente dicho, como así también los resultados de tal proceso, siendo estos últimos verdaderos indicadores de la calidad de la educación. ${ }^{6}$ Ahora, en cuanto a la gestión financiera, se debe destacar que el concepto de accountability educacional recoge del ámbito financiero la responsabilidad que le cabe a toda persona a quien se le confía dinero informar respecto de todo desembolso de fondos, entregar una "cuenta" de cómo se utilizaron dichos fondos y qué se logró con ellos. ${ }^{7}$ En términos concretos, para el caso de Chile, dar cuenta de la redituabilidad económica y social de los recursos públicos invertidos en educación.

En la educación superior en Chile, los logros o resultados obtenidos se han evaluado fundamentalmente en datos, tales como: tasas de aprobación, repetición, abandono, graduación y de continuidad hacia niveles educativos superiores, que indicarían en alguna medida el nivel de desempeño de las instituciones educativas. ${ }^{8}$ Esta forma de medir los resultados de la educación superior en Chile, al menos a la luz de los antecedentes expuestos, no contempla el impacto del trabajo calificado en la productividad y crecimiento económico del país ${ }^{9}$, así como tampoco el aumento de las remuneraciones asociadas a dicho trabajo calificado. ${ }^{10}$

Por ello, el presente estudio analiza cuantitativamente las variables productividad y remuneraciones para el trabajo calificado, correlacionándolas acorde a las hipótesis planteadas en la literatura económica. ${ }^{11}$ Fundamentalmente, en lo que respecta a sus relaciones de causalidad y dependencia ${ }^{12}$, que establecen que el capital humano permite aumentar la productividad, y que una mayor productividad permite aumentar las remuneraciones.

\section{MATERIAL Y MÉTODOS}

La presente investigación tiene como principal objetivo contrastar empíricamente para el caso de Chile dos de los postulados más recurrentes en la economía de la educación, principalmente, en lo relacionado con capital humano avanzado o trabajo calificado. ${ }^{13}$ El primero de ellos plantea la siguiente hipótesis: que mientras mayor sea el capital humano de un país, mayor será su productividad; y, el segundo, señala como hipótesis: que la mayor productividad del trabajo calificado es recompensada o "premiada" con un mayor nivel de remuneraciones. Algunos estudios públicos ${ }^{14}$ dan cuenta que estas relaciones de causalidad y efecto parecen ser muy coherentes y atingentes a la economía chilena, sin embargo, no existen estudios que hayan podido constatar cabalmente dichas relaciones en el largo plazo.
Para constatar dichas hipótesis, el estudio se presenta bajo una tipología correlacional/causal, pretendiendo analizar y evaluar la relación de largo plazo entre las variables Productividad y Remuneraciones para el Trabajo Calificado. Esto implica un análisis cuantitativo de las variables bajo estudio. En tal sentido, se definirán las variables explicadas y las explicativas para las series temporales de datos que van desde el año 1990 al 2009. Las herramientas estadísticas a utilizar para trabajar las series serán la regresión y la cointegración. La primera, permitirá correlacionar las variables, medir su impacto y evaluar cuán determinante es la relación económica planteada. La segunda, contribuiría a determinar para el largo plazo la existencia o no de una regresión espuria. Lo que daría cuenta si las series relacionadas están o no cointegradas. ${ }^{15}$ Para facilitar el análisis cuantitativo de los datos y sus relaciones se utilizará el software Excel y Eviews.

Las fuentes de información serán de tipo secundaria, confiable y validada, provenientes, fundamentalmente, de instituciones de gobierno. Específicamente, se utilizarán estadísticas oficiales de organismos tales como el Instituto Nacional de Estadísticas (INE), el Banco Central de Chile (BCCH), el Ministerio de Educación (MINEDUC), el Ministerio de Desarrollo Social (Ex-MIDEPLAN) y el Ministerio de Hacienda. Adicionalmente, se utilizarán documentos y publicaciones de reconocido prestigio.

\section{RESULTADOS}

\section{Relación entre la Productividad y el Trabajo Calificado}

Una de las mayores premisas asumidas actualmente a nivel internacional es que la inversión en capital humano posibilita un mayor crecimiento económico, fundamentalmente debido a la mejora en la productividad del trabajo. Esto, en razón de la adquisición de nuevos conocimientos, capacidades y habilidades, que configuran cierto stock de capital humano que se acumula con el tiempo acorde a la velocidad de los aprendizajes. ${ }^{16}$ Relevando al factor capital humano incluso por encima del factor capital físico,

Tabla 1.

Variación de la PT y del TC. Chile 1990-2009.

\begin{tabular}{lcccc} 
Años & PT & $\Delta$ PT & TC & $\Delta$ TC \\
\hline 1990 & 0,65 & & 11,000 & \\
1991 & 5,43 & 7,31 & 11,500 & 0,0455 \\
\hline 1992 & 7,33 & 0,35 & 12,000 & 0,0435 \\
1993 & 0,93 & $-0,87$ & 12,650 & 0,0542 \\
\hline 1994 & 2,53 & 1,72 & 14,165 & 0,1198 \\
1995 & 6,69 & 1,65 & 14,239 & 0,0052 \\
\hline 1996 & 3,13 & $-0,53$ & 16,457 & 0,1558 \\
1997 & 2,22 & $-0,29$ & 17,342 & 0,0538 \\
1998 & $-0,99$ & $-1,45$ & 20,285 & 0,1697 \\
1999 & $-1,80$ & 0,82 & 22,387 & 0,1036 \\
\hline 2000 & 1,39 & $-1,77$ & 19,491 & $-0,1294$ \\
2001 & 0,86 & $-0,38$ & 21,069 & 0,0810 \\
\hline 2002 & $-0,68$ & $-1,79$ & 23,386 & 0,1100 \\
\hline 2003 & $-0,07$ & $-0,90$ & 22,240 & $-0,0490$ \\
\hline 2004 & 2,48 & $-38,33$ & 21,085 & $-0,0519$ \\
2005 & 0,65 & $-0,74$ & 28,949 & 0,3730 \\
\hline 2006 & 1,07 & 0,64 & 29,597 & 0,0224 \\
2007 & 0,21 & $-0,80$ & 30,671 & 0,0363 \\
2008 & $-1,37$ & $-7,44$ & 34,804 & 0,1348 \\
\hline 2009 & $-3,43$ & 1,50 & 37,362 & 0,0735 \\
\hline Promedio & & $-2,17$ & & 0,0711 \\
\hline
\end{tabular}

Fuente: Elaboración Propia en base a datos del MINEDUC 2012 y del BCCH. 
lo cual rompe las teorías clásicas del crecimiento económico, como las expuestas en Solow. ${ }^{17}$ En este contexto, el trabajo calificado pasa a ser el factor de producción más importante de la empresa, posibilitando el aumento sostenido de la productividad.

Para constatar tal significativo planteamiento se procede, en primer lugar, a realizar un análisis de series de tiempo para la relación entre las variables Productividad (PT) y Trabajo Calificado (TC), determinando las variaciones anuales para cada una ellas. La siguiente tabla muestra la variación de la Productividad $(\Delta \mathrm{PT})$ y la variación del Trabajo Calificado $(\Delta \mathrm{TC})$. Se observa que para el período bajo estudio, la PT ha tenido un comportamiento bastante disímil con aumentos y disminuciones. En particular, se aprecia una tendencia hacia un comportamiento negativo en los últimos años, arrojando una variación promedio anual de $-2,17$. Por el contrario, el TC ha tenido un crecimiento permanente para casi todo el período analizado, presentando una variación promedio anual positiva. Lo cual podría interpretarse como una disociación entre el comportamiento de ambas variables, lo cual descartaría una relación directa entre el TC y la PT. Menos aún, se podría asumir alguna relación de causalidad.

Lo anterior se puede corroborar al hacer el ejercicio de determinar la correlación entre las series de variaciones de ambas variables. ${ }^{18}$ La Tabla 2 muestra los resultados. Al hacer este cálculo se obtiene un coeficiente de correlación de sólo 0,235. Lo que indicaría una cierta relación positiva entre la PT y el TC, pero infima, por lo que no se podría inferir una relación directa ni menos de causalidad. Se observa que el coeficiente de determinación es bajísimo con un 0,055 e, incluso, este mismo coeficiente ajustado es negativo. Lo que daría cuenta que la PT no se explicaría por el TC del país.

Para indagar más aun la relación entre las variables, se realiza adicionalmente un análisis de regresión, considerando que la variable dependiente es la PT y la variable independiente es el TC. Complementariamente, se realiza un análisis de cointegración para evaluar la relación entre las series PT y TC en el largo plazo.

Tabla 2.

Resumen Estadísticas de Correlación. PT y TC.

\begin{tabular}{lc} 
Resumen & \\
Coeficiente de correlación múltiple & 0,234946456 \\
Coeficiente de determinación R^2 & 0,055199837 \\
\hline R`2 ajustado & $-0,000376643$ \\
Error típico & 9,158052962 \\
\hline Observaciones & 19
\end{tabular}

Fuente: Reporte Software Excel.

Tabla 3.

Resumen Regresión. Productividad - Trabajo Calificado.

$\begin{array}{lcccc}\text { Variable } & \text { Coefficient } & \text { Std. Error } & \text { t-Statistic } & \text { Prob. } \\ \text { C } & -3,628781 & 2,560979 & -1,416951 & 0,1746 \\ \text { TC } & 20,63197 & 20,75791 & 0,993933 & 0,3342 \\ \text { R-squared } & 0,054920 & \text { Mean dependent var } & -2,173684 \\ \text { Adjusted R-squared } & -0,000673 & \text { S.D. dependent var } & 9,156189 \\ \text { S.E. of regression } & 9,159267 & \text { Akaike info criterion } & 7,366710 \\ \text { Sum squared resid } & 1426,167 & \text { Schwarz criterion } & 7,466125 \\ \text { Log likelihood } & -67,98375 & \text { Hannan-Quinn criter. } & 7,383535 \\ \text { F-statistic } & 0,987903 & \text { Durbin-Watson stat } & 1,851646 \\ \text { Prob(F-statistic) } & 0,334193 & & \end{array}$

Dependent Variable: PT, Method: Least Squares, Date: 08/23/13 Time: 12:03 Sample: 1991 2009, Included observations: 19

Fuente: Reporte Regresión Software Eviews 7.
Gráfico 1.

Comportamiento Residuos Regresión. Productividad-Trabajo Calificado.

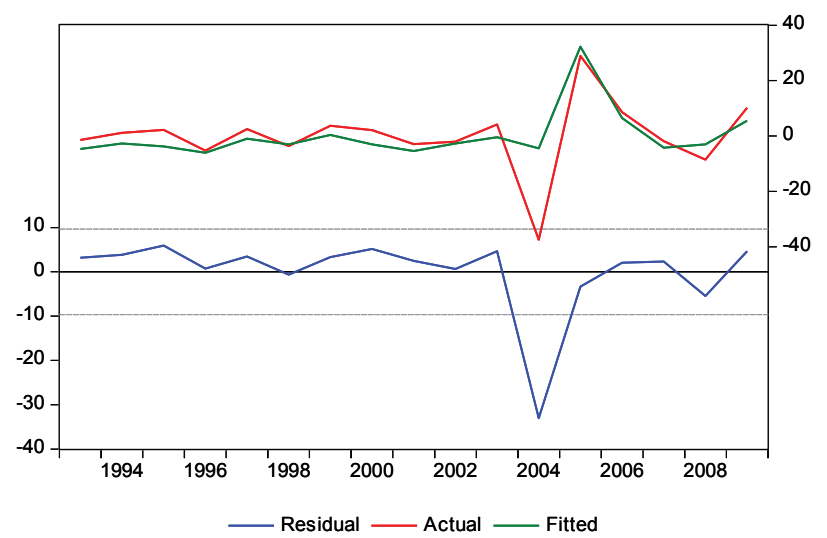

Fuente: Reporte Residuos Software Eviews 7.

La siguiente tabla resumen (Tabla 3) muestra los resultados del análisis de regresión vía Eviews. Se puede observar que la tau calculada no es significativa con un valor de 0,993933, dado que tiene una probabilidad asociada superior a 0,05. Aunque el parámetro de sensibilidad de la variable explicativa (TC) es de 20,63 lo que indicaría que frente a un aumento del TC la productividad aumenta considerablemente. Pero esta conclusión no tiene soporte de significatividad.

Ahora, si se observa la gráfica de los residuos no se aprecia una tendencia creciente, sino más bien plana y con cierta oscilación que podria dar cuenta de una serie estacionaria.

Tabla 4.

Resumen Prueba Interna Raíz Unitaria. Test Valores Críticos.

Productividad Total de los Factores-Capital Humano Avanzado.

\begin{tabular}{|c|c|c|c|c|}
\hline \multicolumn{5}{|c|}{ Null Hypothesis: $R$ has a unit root } \\
\hline \multicolumn{5}{|l|}{$\begin{array}{l}\text { Exogenous: Constant } \\
\text { LagLength: } 1 \text { (Fixed) }\end{array}$} \\
\hline Test criticalvalues: & $\begin{array}{c}\text { er test statisti } \\
1 \% \text { level } \\
5 \% \text { level } \\
10 \% \text { level }\end{array}$ & & $\begin{array}{c}\text { t-Statistic } \\
-2,939680 \\
-3,886751 \\
-3,052169 \\
-2,666593\end{array}$ & $\begin{array}{l}\text { Prob.* }^{*} \\
0,0615\end{array}$ \\
\hline \multicolumn{5}{|c|}{$\begin{array}{l}\text { *MacKinnon (1996) one-sided p-values. } \\
\text { Warning: Probabilities and critical values cal } \\
\text { and may not be accurate for a sample size } 0 \\
\text { Augmented Dickey-Fuller Test Equation } \\
\text { Dependent Variable: } D(R) \\
\text { Method: LeastSquares } \\
\text { Date: } 08 / 23 / 13 \text { Time: } 12: 10 \\
\text { Sample (adjusted): } 19932009 \\
\text { Includedobservations: } 17 \text { afteradjustments }\end{array}$} \\
\hline Variable & Coefficient & Std. Error & t-Statistic & Prob. \\
\hline $\mathrm{R}(-1)$ & $-1,066085$ & 0,362654 & $-2,939680$ & 0,0108 \\
\hline $\mathrm{D}(\mathrm{R}(-1))$ & 0,076895 & 0,260761 & 0,294887 & 0,7724 \\
\hline C & $-0,746928$ & 2,347422 & $-0,318191$ & 0,7550 \\
\hline R-squared & 0,499096 & Mean depe & tvar0,031238 & \\
\hline Adjusted R-squared & 0,427538 & S.D. depenc & $\operatorname{ar} 12,73560$ & \\
\hline S.E. of regression & 9,635906 & Akaikeinfoc & ion7,527655 & \\
\hline Sum squaredresid & 1299,910 & Schwarzer & n7,674693 & \\
\hline Log likelihood & $-60,98507$ & Hannan- $\mathrm{Ou}_{\mathrm{u}}$ & riter.7,542271 & \\
\hline F-statistic & 6,974733 & Durbin-Wat & stat1,992147 & \\
\hline Prob(F-statistic) & 0,007912 & & & \\
\hline
\end{tabular}

Fuente: Reporte Regresión Software Eviews 7. 
Por lo tanto, para corroborar si la serie es estacionaria o no, se ejecuta la prueba interna del Eviews, de forma tal de comparar la tau calculada con los valores críticos de las tablas de Mackinnon. ${ }^{19}$ Se observa en Tabla 4 que, en términos absolutos, la tau calculada es menor a los valores críticos analizados, excepto para un nivel del 10\% (pero está muy cercano a él). Para los primeros niveles (1\% y 5\%) dado que la tau está bajo los valores críticos de referencia, se acepta la hipótesis nula, es decir, existe raíz unitaria, las series son estacionarias no hay cointegración. Por lo tanto, no hay una relación de largo plazo entre la PT y el TC.

\section{Evolución de las Remuneraciones del Trabajo Calificado y No Calificado}

La relación de las remuneraciones con el tipo de calificación del trabajo ha sido ampliamente aceptada, formulándose incluso diversas ecuaciones de ingreso que reconocen la relación directa entre ambas variables. ${ }^{20}$ Para constatar tal relación, la Tabla 5 muestra la variación porcentual que ha tenido el índice de remuneraciones, tanto para el trabajo calificado, como no calificado en Chile. Observándose un comportamiento muy similar entre

Tabla 5.

Evolución \% del IR Trabajo Calificado y No Calificado. Chile 1993-2009.

\begin{tabular}{lccc} 
Años & $\begin{array}{c}\Delta \% \text { IR Trabajo } \\
\text { Calificado }\end{array}$ & $\begin{array}{c}\Delta \% \text { IR Trabajo } \\
\text { No Calificado }\end{array}$ & $\begin{array}{c}\Delta \% \text { IR } \\
\text { Total }\end{array}$ \\
\hline 1993 & $18,7 \%$ & $14,1 \%$ & $15,1 \%$ \\
1994 & $14,6 \%$ & $14,0 \%$ & $14,6 \%$ \\
1995 & $15,8 \%$ & $15,2 \%$ & $13,7 \%$ \\
1996 & $12,8 \%$ & $9,3 \%$ & $9,5 \%$ \\
1997 & $9,1 \%$ & $8,3 \%$ & $7,4 \%$ \\
1998 & $10,6 \%$ & $7,1 \%$ & $7,7 \%$ \\
1999 & $7,0 \%$ & $1,3 \%$ & $4,7 \%$ \\
2000 & $4,7 \%$ & $5,3 \%$ & $5,3 \%$ \\
\hline 2001 & $6,9 \%$ & $5,9 \%$ & $5,3 \%$ \\
2002 & $3,6 \%$ & $3,7 \%$ & $4,0 \%$ \\
2003 & $2,9 \%$ & $4,9 \%$ & $3,4 \%$ \\
2004 & $2,9 \%$ & $1,4 \%$ & $2,7 \%$ \\
2005 & $5,2 \%$ & $7,0 \%$ & $6,3 \%$ \\
\hline 2006 & $5,7 \%$ & $5,4 \%$ & $5,7 \%$ \\
\hline 2007 & $7,5 \%$ & $8,0 \%$ & $8,3 \%$ \\
2008 & $8,8 \%$ & $8,1 \%$ & $8,5 \%$ \\
2009 & $6,3 \%$ & $4,4 \%$ & $5,0 \%$ \\
Promedio & $8,4 \%$ & $7,3 \%$ & $7,5 \%$ \\
\hline
\end{tabular}

Fuente: Elaboración propia en base a datos del INE.

\section{Gráfico 2.}

Evolución \% del IR Trabajo Calificado y No Calificado. Chile 1993-2009.

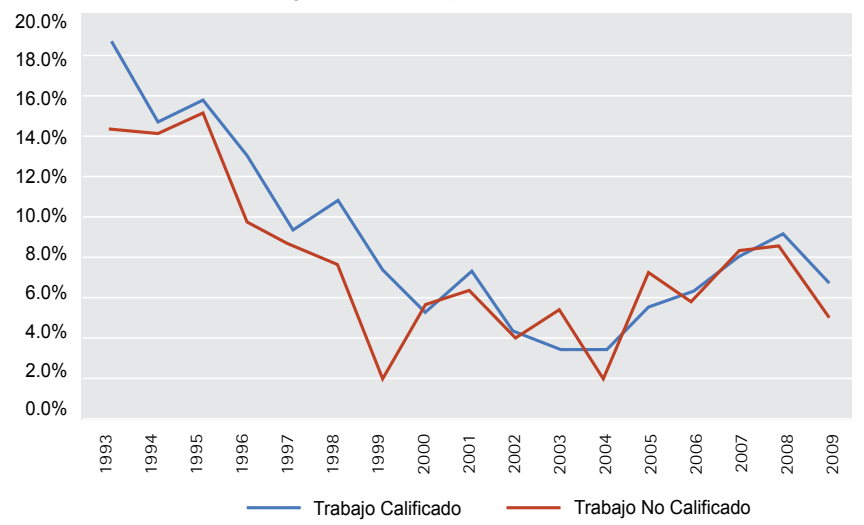

Fuente: Elaboración propia en base a datos del INE. ambos índices. Con una variación promedio anual entre un $7 \%$ y 8\%. Con un fuerte crecimiento entre los años 1993-1996, teniendo un promedio anual en torno al 14\%. Para luego decaer paulatinamente entre los años 1997-2009.

La gráfica entre ambos índices es clarificadora de lo homogéneo del comportamiento evolutivo de las remuneraciones, no existiendo mayor diferencia entre Trabajo Calificado y No Calificado. (Ver gráfico 2).

No obstante, esta aparente homogeneidad en la evolución de las remuneraciones, la situación cambia totalmente si consideramos ahora los diferenciales de ingresos. Evidenciándose una evidente desigualdad entre el nivel de ingreso de los Trabajadores Calificados y el de los No Calificados. Esto se puede constatar a través del cálculo de la razón entre las remuneraciones de los profesionales y las demás ocupaciones para obtener un indicador que dé cuenta del diferencial de remuneraciones: mientras mayor sea esta razón, mayor será el diferencial de remuneración favorable al grupo de profesionales. Claro está, la distribución de ingresos la realiza el mercado con todas las imperfecciones que ello conlleva y que no pueden ser resueltas por sí mismas. ${ }^{21}$

En particular, se observa (Tabla 6) un aumento de la brecha entre los profesionales y los trabajadores no calificados. Para el año 1993, la remuneración de un profesional era 3,11veces superior a la de un trabajador no calificado. En cambio, en el año 2009 el diferencial de remuneraciones favorable a los profesionales fue de 4,64 veces superior a la de un trabajador no calificado, lo que deja en evidencia el creciente "premio" otorgado por el mercado laboral al trabajador calificado lo que, sin duda, ha incentivado la demanda por carreras profesionales en Chile, fundamentalmente universitarias, tal como se menciona en Bruner y Elacqua ${ }^{22}$ y en Bruner $^{23}$, con un crecimiento explosivo de las matrículas en educación superior.

En definitiva, las remuneraciones de los profesionales no sólo son altas, comparativamente hablando, sino que han crecido notablemente durante el período bajo estudio. En este sentido, el aumento del "premio" de ser profesional observado en Chile ya lo habían experimentado países hoy en día desarrollados ${ }^{24}$, dando cuenta de un fenómeno recurrente en las etapas previas al

Tabla 6.

Razón de Remuneraciones de Profesionales Respecto a los demás Grupos. Chile 1993-2009.

\begin{tabular}{ccccc} 
Años & General & Técnicos & $\begin{array}{c}\text { Personal } \\
\text { Administrativo }\end{array}$ & $\begin{array}{c}\text { Trabajadores } \\
\text { No Calificados }\end{array}$ \\
\hline 1993 & 2,25 & 1,392 & 1,92 & 3,11 \\
1994 & 2,17 & 1,430 & 1,82 & 3,08 \\
1995 & 2,30 & 1,468 & 1,85 & 3,14 \\
1996 & 2,38 & 1,490 & 1,91 & 3,27 \\
1997 & 2,44 & 1,550 & 1,94 & 3,33 \\
1998 & 2,60 & 1,612 & 2,10 & 3,62 \\
1999 & 2,59 & 1,596 & 2,01 & 3,74 \\
2000 & 2,63 & 1,572 & 3,68 & 3,74 \\
2001 & 2,70 & 1,601 & 2,14 & 3,91 \\
2002 & 2,70 & 1,587 & 2,13 & 3,91 \\
2003 & 2,77 & 1,637 & 2,22 & 3,99 \\
2004 & 2,80 & 1,635 & 2,21 & 4,18 \\
2005 & 2,79 & 1,657 & 2,21 & 4,12 \\
2006 & 2,79 & 1,833 & 2,46 & 4,57 \\
2007 & 2,74 & 1,790 & 2,36 & 4,54 \\
2008 & 2,79 & 1,830 & 2,38 & 4,65 \\
\hline 2009 & 2,80 & 1,797 & 2,36 & 4,64 \\
\hline
\end{tabular}

Fuente: Elaboración propia en base a datos del INE. 
desarrollo. Si bien es cierto, en estos países aún se mantienen diferenciales de remuneraciones a favor de profesionales, el guarismo es bastante menor respecto de décadas atrás que, por ejemplo, para el caso de Estados Unidos de América, las remuneraciones de los profesionales no sobrepasan en dos veces las de los trabajadores no calificados. ${ }^{25}$

Ahora, en vista de estos antecedentes y algunos estudios empíricos ${ }^{26}$, es de toda lógica inferir que el gran aumento de las remuneraciones de profesionales en Chile ha provocado a su vez el gran aumento de la oferta de este tipo de trabajadores. Cabe recordar que los titulados de las Instituciones de Educación Superior el año 1999 era de sólo 35.771; en cambio, el año 2011 alcanzan los 122.194 profesionales.

\section{Productividad del Trabajo Calificado y las Remuneraciones}

Otro de los grandes postulados de la economía de la educación se refiere a que la mayor productividad del trabajo calificado debe traer consigo el justo "premio" de una mayor remuneración. Esta relación ha sido contrastada empíricamente en el presente estudio, observándose (Gráfico 3) que existen varios períodos en que la variación de las remuneraciones del trabajo calificado no es acompañada coherentemente por la variación de la productividad, como lo sugiere la literatura económica. ${ }^{27}$ Por ejemplo, para el año 1997 la productividad de la educación superior fue negativa de -0,64; en cambio, para ese mismo año el índice de remuneraciones creció un 9,15\%. Similar situación sucede para el año 1998, con una productividad de la educación superior negativa de -0,36 y una variación positiva del índice de remuneraciones de un 10,63\%. Esto se vuelve a repetir en forma más pronunciada aún para los años 2004 y 2011.

Para agudizar la apreciación de la relación entre la Productividad de la Educación Superior (PES) y las Remuneraciones asociadas ${ }^{28}$, se realiza un análisis de regresión y cointegración, considerando como variable dependiente o explicada, la variación del índice de remuneraciones del trabajo calificado, y como variable independiente o explicativa, la productividad de la educación superior.

La siguiente tabla resumen (Tabla 7) muestra los resultados del análisis de regresión vía Eviews. Se puede observar que la tau calculada no es significativa con un valor de 1.256, dado que tiene una probabilidad superior a 0,05. Además presenta un coeficiente de determinación bajo que no supera el 0,08. Esto

\section{Gráfico 3.}

Productividad Educación Superior versus Variación IR Trabajo Calificado. Chile 1993-2012.

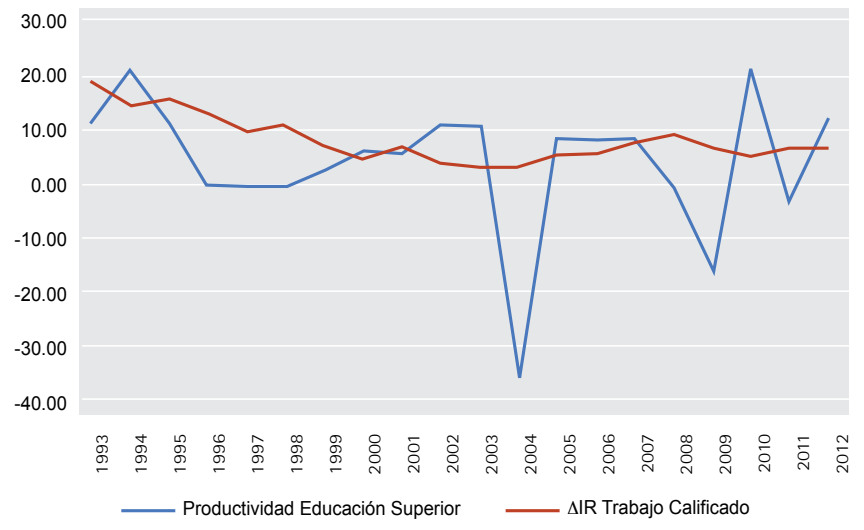

Fuente: Elaboración propia en base a datos del INE y del MINEDUC 2012.
Tabla 7.

Resumen Regresión. Productividad Educación Superior-Remuneraciones Trabajo Calificado.

Dependent Variable: IR

Method: Least Squares

Date: 10/09/13 Time: 17:31

Sample: 19932012

Included observations: 20

$\begin{array}{lcccc}\text { Variable } & \text { Coefficient } & \text { Std. Error } & \text { t-Statistic } & \text { Prob. } \\ \text { C } & 7,624198 & 1,023100 & 7,452054 & 0,0000 \\ \text { PES } & 0,102510 & 0,081597 & 1,256290 & 0,2251 \\ \text { R-squared } & 0,080613 & \text { Mean dependent var } & 8,024500 \\ \text { Adjusted R-squared } & 0,029536 & \text { S.D. dependent var } & 4,413550 \\ \text { S.E. of regression } & 4,347882 & \text { Akaike info criterion } & 5,871894 \\ \text { Sum squared resid } & 340,2734 & \text { Schwarz criterion } & 5,971468 \\ \text { Log likelihood } & -56,71894 & \text { Hannan-Quinn criter. } & 5,891332 \\ \text { F-statistic } & 1,578265 & \text { Durbin-Watson stat } & 0,503960 \\ \text { Prob(F-statistic) } & 0,225072 & & \end{array}$

Fuente: Reporte Regresión Software Eviews 7.

Gráfico 4.

Comportamiento Residuos.

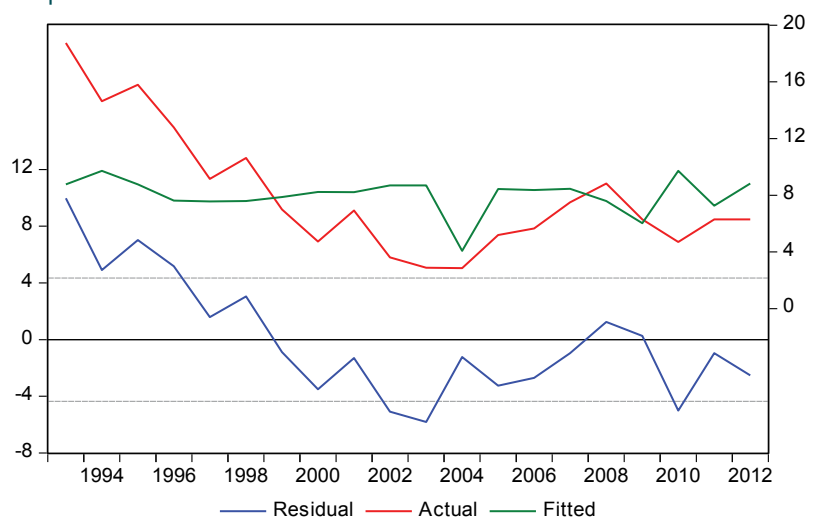

Fuente: Reporte Residuos Software Eviews 7.

Tabla 8.

Resumen Regresión Primera Diferencia Residuos. Productividad Educación Superior-Remuneraciones Trabajo Calificado.

\section{Dependent Variable: DR}

Method: LeastSquares

Date: 10/08/13 Time: 16:37

Sample (adjusted): 19952012

Includedobservations: 18 afteradjustments

$\begin{array}{lcccc}\text { Variable } & \text { Coefficient } & \text { Std. Error } & \text { t-Statistic } & \text { Prob. } \\ \text { C } & -0,728278 & 0,616097 & -1.182084 & 0,2556 \\ \text { R1 } & -0,327534 & 0,170016 & -1,926496 & 0,0732 \\ \text { DR1 } & -0,295793 & 0,202915 & -1,457719 & 0,1655 \\ \text { R-squared } & 0,315072 & \text { Mean dependentvar } & -0,412947 \\ \text { Adjusted R-squared } & 0,223748 & \text { S.D. dependentvar } & 2,897941 \\ \text { S.E. of regression } & 2,553236 & \text { Akaikeinfocriterion } & 4,863612 \\ \text { Sum squaredresid } & 97,78523 & \text { Schwarzcriterion } & 5,012007 \\ \text { Log likelihood } & -40,77251 & \text { Hannan-Quinncriter. } & 4,884074 \\ \text { F-statistic } & 3,450049 & \text { Durbin-Watson stat } & 2,107984 \\ \text { Prob(F-statistic) } & 0,058525 & & \end{array}$

Fuente: Reporte Regresión Software Eviews 7.

indicaría que la variable PES explicaría sólo en un 8\% la variación de las remuneraciones. Por su parte, el parámetro de sensibilidad de la variable explicativa (PES) es de 0,102510, lo que indicaría que frente a un aumento de la PES el nivel de remuneraciones podría aumentar levemente. Esto es, por ejemplo, si la PES aumentara en un 10\%, las remuneraciones aumentarían sólo 
un 1\%. Antecedentes empíricos que se distancian de algunos estudios que sugieren mayores tasas de rentabilidad para el capital humano avanzado. $^{29}$

Ahora, si se observa la Gráfica 4 de los residuos no se aprecia una determinada tendencia sino más bien, la curva presenta un comportamiento oscilante que podría dar cuenta de una serie estacionaria. Para determinar la estacionariedad de la serie se aplica el análisis de cointegración con el cual se obtiene a continuación la primera diferencia de los residuos (DR).

La Tabla 8 muestra los resultados de la regresión de la primera diferencia de los residuos de la regresión entre la productividad de la educación superior y las remuneraciones del trabajo calificado, arrojando una tau de 1,92 que al compararse con los parámetros de Mackinnon resulta ser un valor no significativo, como se muestra en Tabla 9. Por lo tanto, las series no estarían cointegradas.

Lo anterior, se puede corroborar con la prueba interna del Eviews, de forma tal de comparar la tau calculada con los valores críticos de las Tablas de Mackinnon.

Se observa en Tabla 9 que, en términos absolutos, la tau calculada es menor a los valores críticos analizados. Dado que la tau está bajo los valores críticos de referencia, se acepta la hipótesis nula, es decir, existe raíz unitaria, las series son estacionarias, no están cointegradas. Por lo tanto, no hay una relación de largo plazo entre la PES y el IR.

\section{CONCLUSIONES}

La investigación posibilitó contrastar empíricamente dos grandes hipótesis de la literatura económica respecto del trabajo-capital humano y su correspondiente remuneración:

Hipótesis 1: Mientras mayor sea el capital humano de un país, mayor será su productividad.

Hipótesis 2: La mayor productividad del trabajo calificado es recompensada o "premiada" con un mayor nivel de remuneraciones.

Respecto a la primera hipótesis, realizando un análisis de relación entre las variables se observó una correlación levemente positiva de 0,2235 . No pudiéndose inferir una relación directa y menos de causalidad, con un coeficiente de determinación bajísimo de 0,055. Además, los residuos de las series presentan raíz unitaria, por lo cual las series no estarían cointegradas, lo que implica que no existe, para el caso de Chile, una relación de largo plazo entre la productividad total de país y el trabajo calificado. Este hallazgo es muy relevante, dada la hipótesis recurrentemente aceptada de que un mayor trabajo calificado genera mayor productividad en el país, no sería del todo válida, al menos en el caso de Chile. Debiéndose quizás cuestionar, el tipo y calidad del capital humano avanzado que se está formando.

Respecto a la segunda hipótesis, se evidenció para varios subperíodos, respecto del período de investigación, que la variación de las remuneraciones del trabajo calificado no es acompañada coherentemente por la variación de la productividad, como lo siguiere la literatura económica.

Es más, al realizar un análisis de regresión y cointegración, considerando como variable explicada la variación del índice de remuneraciones del trabajo calificado, y como variable explicativa, la productividad del gasto público en educación superior, se pudo determinar que el parámetro para la variable explicativa es de sólo 0,10 . Lo que indicaría que un aumento de un 10\% de la productividad en educación superior aumentaría sólo en un 1\% las remuneraciones del

\section{Tabla 9.}

Resumen Prueba Interna Raíz Unitaria. Test Valores Críticos. Productividad Educación Superior-Remuneraciones Trabajo Calificado.

\begin{tabular}{|c|c|c|c|c|}
\hline \multicolumn{5}{|c|}{ Null Hypothesis: PI has a unit root } \\
\hline \multicolumn{5}{|l|}{$\begin{array}{l}\text { Exogenous: Constant } \\
\text { Lag Length: } 1 \text { (Fixed) }\end{array}$} \\
\hline & & & t-Statistic & Prob.* \\
\hline \multicolumn{3}{|c|}{ Augmented Dickey-Fuller test statistic } & $-1,926496$ & 0,3137 \\
\hline \multirow[t]{3}{*}{ Test critical values: } & $1 \%$ level & & $-3,857386$ & \\
\hline & $5 \%$ level & & $-3,040391$ & \\
\hline & $10 \%$ level & & $-2,660551$ & \\
\hline \multicolumn{5}{|c|}{ *MacKinnon (1996) one-sided p-values. } \\
\hline \multicolumn{5}{|c|}{$\begin{array}{l}\text { Warning: Probabilities and critical values } \\
\text { and may not be accurate for a sampl }\end{array}$} \\
\hline \multicolumn{5}{|c|}{ Augmented Dickey-Fuller Test Equation } \\
\hline \multicolumn{5}{|c|}{ Dependent Variable: D(PI) } \\
\hline \multicolumn{5}{|c|}{ Method: Least Squares } \\
\hline \multicolumn{5}{|c|}{ Date: 10/10/13 Time: 11:28 } \\
\hline \multicolumn{5}{|c|}{ Sample (adjusted): 19952012} \\
\hline \multicolumn{5}{|c|}{ Included observations: 18 after adjustments } \\
\hline Variable & Coefficient & Std. Error & t-Statistic & Prob. \\
\hline $\operatorname{PI}(-1)$ & $-0,327534$ & 0,170016 & $-1,926496$ & 0,0732 \\
\hline $\mathrm{D}(\mathrm{PI}(-1))$ & $-0,295793$ & 0,202915 & $-1,457719$ & 0,1655 \\
\hline C & $-0,728278$ & 0,616097 & $-1,182084$ & 0,2556 \\
\hline R-squared & 0,315072 & Mean $d$ & dent var & $-0,412947$ \\
\hline Adjusted R-squared & 0,223748 & S.D. de & dent var & 2,897941 \\
\hline S.E. of regression & 2,553236 & Akaike & criterion & 4,863612 \\
\hline Sum squared resid & 97,78523 & Schws & riterion & 5,012007 \\
\hline Log likelihood & $-40,77251$ & Hannan & inn criter. & 4,884074 \\
\hline F-statistic & 3,450049 & Durbin- & tson stat & 2,107984 \\
\hline Prob(F-statistic) & 0,058525 & & & \\
\hline
\end{tabular}

Fuente: Reporte Regresión Software Eviews 7.

trabajo calificado. Además, el coeficiente de determinación es de sólo 0,08 , lo que implica que la variable productividad del gasto público en educación superior sólo explicaría en un $8 \%$ la variación de las remuneraciones de trabajo calificado. Adicionalmente se pudo determinar, a través del análisis de cointegración, que no existe una relación de largo plazo entre la productividad en educación superior y las remuneraciones del trabajo calificado.

No obstante lo anterior, se presenta para el período bajo estudio un aumento significativo de la brecha de ingresos entre los Trabajadores Calificados (profesionales) y los No Calificados. Es así como, mientras en 1993 la brecha de remuneración a favor de un profesional era de 3,11 veces respecto a un trabajador No Calificado, el año 2009 dicha brecha es favorable a los profesionales en 4,64 veces. Esto da cuenta de la valoración del mercado laboral respecto del trabajador calificado, lo que ha motivado fuertemente la demanda de carreras profesionales en Chile, especialmente universitarias. Aun cuando, en términos de productividad, no existe empíricamente una relación económica coherente a los postulados de la economía de la educación. Esta situación también ha sido evidenciada en otros países de Latinoamérica con niveles de desarrollo similares a Chile $\mathrm{e}^{30}$, incluso durante períodos de depresión económica. Observándose para estos períodos un aumento de la brecha de ingresos entre trabajadores calificados respecto de aquellos sin mayor calificación. Concluyéndose que las normativas de selección privilegian en Latinoamérica la certificación educacional, en coherencia con la teoría credencialista de Collins. ${ }^{31}$ En tal sentido, y como futura línea de investigación, sería muy interesante contrastar empíricamente ésta teoría para el caso de Chile. 
1. Beyer H. Educación y Desigualdad de Ingresos: Una Nueva Mirada. Santiago: Centro de Estudios Públicos; 1999. (Documento de Trabajo; 297).

2. Casassús J. Acerca de la calidad de la educación. Ponencia para el teleseminario sobre calidad de la educación. Santiago de Chile: UNESCO-Oficina Regional de Educación; 1995.

3. Giménez G. La dotación de capital humano en América Latina y el Caribe. Revista CEPAL. 2005;(86):103-122.

4. Peñaranda A. Educación, Experiencia e Ingresos en el Sector Informal en Colombia. Revista Academia de Medicina [en línea] 2004;26(2)[citado may 2014]. Disponible en: http://www.encolombia.com/medicina/academedicina/ academ26265-educacion2.htm

5. Chile. SIES. Informe sobre la Evolución de la Matricula en la Educación Superior en Chile. Periodo 1990-2009. Santiago: MINEDUC -División de Educación Superior; 2010.

6. Shupe D. Productivity, Quality, and Accountability in Higher Education. J Contin Higher Educ. 2009;47(1):2-13.

7. PREAL-CIDE. Accountability Educacional; Posibilidades y Desafios para América Latina a partir de la Experiencia Internacional. Santiago: San Marino; 2006.

8. Bernasconi A, Rojas F. Informe sobre la Educación Superior en Chile: 1980-2003. Caracas: UNESCO-IESAL; 2003. (IES/2003/ED/PI/55).

9. Lucas R. On the Mechanics of Economic Development. J Monetary Econ.1988; 22(1):3-42.

10. Mcmahon W W. The impact of human capital on non-market outcomes and feedbacks on economic development: International symposium report. In: John F Helliwell, editor. The contribution of human and social capital to sustained economic growth and well-being. Ottawa: Organisation for Economic Cooperation and Development; 2001. p.136-171.

11. Schultz TW. Investment in Human Capital. Am Econ Rev.1961; 51(1):1-17.

12. Becker G. El Capital Humano. Madrid: Alianza; 1983

13. Laroche $M$, Merette $M$, Ruggeri $G$. On the concept and dimensions of human capital in a knowledge-based economy context. Can Public Pol.1999; 25(1):87-100.

14. Chile. MIDEPLAN. Distribución del Capital Humano en Chile. Santiago: MIDEPLAN- Departamento de Competitividad Regional; 2004.

15. Clive G. Análisis de Series Temporales, Cointegración y Aplicaciones. Revista Asturiana de Economia (RAE). 2004; (30):197-206.

16. OCDE. Measuring what people know. Human capital for the knowledge economy. Paris: OECD Publications; 1998.

17. Solow R. A Contribution to the Theory of Economic Growth. $0 \mathrm{~J}$ Econ. 1956;70:65-94.

18. Beyer H. Productividad, Desigualdad y Capital Humano: los Complejos Desafíos de Chile. Santiago: Centro de Estudios Públicos; 2005. (Estudios Públicos; 97)
19. Antúnez-Irgoin C. Análisis De Series De Tiempo. Contribuciones a la Economia[en línea] 2011(feb):1-47[citado may 2014]. Disponible en: http:// www.eumed.net/ce/2011a/chai.htm

20. Willis R. Wage Determinants: a Survey and Reinterpretation of Human Capital Earnings Functions. In: Handbook of Labor Economics. Chicago: University of Chicago and Economic Research Center; 1986.

21. Rodríguez E. La educación superior en Chile y el rol del mercado: ¿culpable o inocente?. Ingeniare. Rev Chil Ing. 2012;20(1):126-135.

22. Brunner JJ, Elacqua G. Informe Capital Humano en Chile. Santiago: Universidad Adolfo lbáñez; 2003.

23. Brunner JJ. Informe sobre la Educación Superior en Chile: Orientando al Mercado. Santiago: Universidad Adolfo lbáñez; 2005.

24. Beyer H. ¿Qué Veinte Años No Es Nada...? Una Mirada a la Desigualdad de Ingresos a partir de las Encuestas CASEN. Santiago: Centro de Estudios Públicos; 2011. (Estudios Públicos; 121)

25. Mizala A, Romaguera P. Remuneraciones y tasa de retorno de los profesionales Chilenos. En: Oferta y demanda de profesionales y técnicos en Chile: El rol de la información pública. Brunner JJ, Meller P, editores. Santiago: Ril; 2004.

26. Sapelli C. Returns to Schooling and Income Distribution by Cohort in Chile: An Analysis Based on Synthetic Panel Data. Santiago: Pontificia Universidad Católica de Chile- Instituto de Economía; 2005. (Documentos de Trabajo; 290).

27. Sapelli C. Ecuaciones de Mincer y las Tasas de Retorno a la Educación en Chile: 1990-1998. Santiago: Pontificia Universidad Católica de ChileInstituto de Economía; 2003. (Documentos de Trabajo; 254).

28. Uribe J, Castellar C. Una Aproximación Econométrica a la Tasa de Retorno Social de la Educación. Revista Sociedad y Economía. 2001;(1):77-102.

29. Urzúa S. La Rentabilidad de la Educación Superior en Chile. Santiago: Centro de Estudios Públicos; 2012. (Estudios Públicos; 125).

30. Merlo JJ. Retornos a la Educación durante una Depresión Económica. Evidencia Empírica para Argentina. [Tesis de Magister]. Santiago: Pontificia Universidad Católica de Chile; 2009.

31. Collins R. La sociedad credencialista; Sociología histórica de la educación y la estratificación. Madrid: Akal; 1989

\section{Referencias complementarias}

Ben-Porath Y. The Production of Human Capital and the Life Cycle of Earnings J Polit Econ. 1967;75(4):352-365.

Chile. MINEDUC. Estudio de la Inversión y de la Gestión Pública en Educación Superior 1990-2011. Santiago: MINEDUC-División Educación Superior; 2012. 